\title{
«Cuando los hechos cambian...» Imágenes futuras de la investigación en educación: entre la continuidad, la salida y la contemplación
}

\author{
"When the facts change...» \\ Future Images of Research in Education: Between Continuity, \\ Exit and Contemplation
}

\section{Rolf Arnold ${ }^{1}$}

\section{Resumen}

Necesitamos un concepto de aprendizaje contemporáneo. Las connotaciones del concepto «formación» o el nexo de «enseñanza» y «aprendizaje» se refieren a una relación tradicional entre educación y tiempo que debe ser problematizada. Los avances de la formación de personas adultas, en cuyo seno la formació se definió como «un proceso cognitivo relacionado con el mundo de vida», con sus desarrollos constructivistas y sistémicos, los avances de las ciencias naturales sobre el yo sináptico, conducen a desvincularnos de los contenidos curriculares y a una teoría formal, que ubica lo biográfico en el centro de lo educativo y postula una formación de la personalidad, un programa que tiene que ver más con las propios rastros emocionales de los seres humanos en sus fases biográficas de desarrollo que con los contenidos de los planes de estudio y currículums.

\section{Palabras claves}

Educación contemporánea, formación de la personalidad, constructivismo sistémico.

\section{Abstract}

We need a concept of contemporary learning. The connotations of the concept «formation» or the nexus of «teaching» and «learning» refer to a traditional relationship between education and time that must be problematized. Advances in the formation of adults, in which the formation was defined as «a cognitive process related to the world of life», with its constructivist and systemic developments, the advances of the natural sciences on the synaptic self, leading to disengagement of the curricular contents and a formal theory, which places the biographical in the center of education and postulates a formation of personality, a program that has more to do with the emotional traces of human beings in their biographical phases of development that with the contents of curricula and study plans..

\section{Keywords}

Contemporary education, personality formation, systemic constructivism. 


\section{Introducción}

El futuro constituye el foco fundamentalísimo del pensamiento pedagógico; aunque también representa sus puntos ciegos. Generalmente la argumentación recae sobre el aumento de lo humano en el tiempo, ya sea como evolución del desarrollo de la competencia y de la identidad del individuo o ya sea como proyecto de género llamado civilización. Este último se define mediante el grado alcanzado históricamente del uso justo de la razón. Solo en contexto de este uso de la razón se puede justificar también qué tipo de formación de personalidad parezca alcanzable y necesaria. Sus formas de expresión se aprecian también en un autodistanciamiento alcanzable por medio del desarrollo de las capacidades, de un analizarse a uno mismo y al mundo de manera sensata y de la fundamentación adecuada de las propias acciones.

Se ha transmitido la siguiente expresión de John Maynard Keynes: «Si los hechos cambian, cambio mi opinión. Y usted, ¿qué hace?» (cita según Chamberland 2015: 191) -una cuestión que nos confronta con nuestra propia práctica del pensamiento y del enjuiciamiento, pero también con nuestro uso del lenguaje y experiencia histórica que en ellos se ha depositado-. Una persona «culta o instruida» es, en este sentido, aquella que es capaz de llevar a cabo un «uso problematizado de la razón» (Ruhloff 1996). Esta interpretación se distancia de toda aquella praxis formativa que fomente un saber, una verdad o un asentimiento unilaterales, sin ninguna sensibilidad por las formas del conocimiento aparente, del desconocimiento o del error. La capacidad de problematizar debe ser aprendida y practicada -en especial la problematización de las propias formas de hablar predilectas, cuyas interpretaciones no son verdaderas por ello, porque las tengamos o porque se expresen en una lengua en la que por casualidad hemos aprendido a pensar-.

\section{Observamos y pensamos en la «prisión de nuestra lengua» (Wittgenstein)}

Precisamente nuestra lengua nos tiene prisioneros con sus nociones - una situación que se hace evidente en alemán, en especial, en el concepto de «Bildung» ${ }^{2}$-. Esta palabra, difícilmente traducible a otras lenguas, va acompañada de connotaciones cuyos orígenes al fin y al cabo tienen carácter religioso: Dios creó al ser humano a su imagen. Por tal motivo, la educación también se concebía como una transformación de la individualidad mediante la cual la intención de Dios - lo divino en cada persona- se expresaba con éxito. Sería interesante investigar estos rastros en el debate educativo alemán más adelante porque estoy seguro que también en otras lenguas -como sucede igualmente en rumano- uno se maneja con términos cargados de connotaciones. Sin embargo, un linguistic turn de tal tipo se quedaría en algo específico, donde se trataría de hacerse entender más allá de las lenguas.

A continuación quiero referirme brevemente al concepto de aprendizaje, que también comienza a destacarse cada vez más en el debate educativo europeo. «Learning - the Treasur within» fue ya el título del informe de la UNESCO del año 1996. Si sometemos al término alemán «Lernen» [aprendizaje] a un análisis etimológico, se constata que dicho término pertenece al grupo de palabras indogermánicas de «leisten» [realizar, lograr] y, entre otras cosas, está relacionado con las palabras. «lehrenı [instruir, enseñar] $\mathrm{y}$ «Lis $\gg$ [lista].

«Bildung» se puede traducir por educación, pero también por cultura, ilustración, formación, desarrollo, crecimiento, instrucción, erudición, conocimientos, creación, composición, etc. «Bilden» deriva de . «Bild», que significa imagen, y también retrato, dibujo, ilustración, pintura, cuadro, aspecto, figura, etc. [N. trad.] 


\begin{abstract}
"Lais" era la denominación de los godos para "ich weißß" [yo sé]. "Lis" es la palabra indogermánica para "gehen" [ir, andar]. Existen claras indicaciones de que ya el aprendizaje temprano se entendía como un proceso en el cual el aprendiz debia transitar un camino y, al mismo tiempo, adquirir conocimiento» ${ }^{3}$
\end{abstract}

En alemán resulta llamativo la proximidad de ambas palabras para Lernen [aprendizaje] y Lehre [enseñanza]; en otras lenguas incluso hay una sola y la misma palabra para ambas actividades: p.e. en griego antiguo existe «didaskein» para ambas palabras: enseñar y aprender:

«El griego original unificó entonces el sentido causal enseñar con el aprendizaje inmediato en una forma [...] como está además en alemán y donde, entre las gentes sencillas, aprender también se dice enseñar». (Riemer 1819: 385).

Así se puede leer en un diccionario manual griego-alemán del año 1819. Sería entonces interesante examinar cómo, mediante la enseñanza, ha podido transformarse cada vez más un aprender como una actividad vital natural (como la respiración) en una acción concreta -hasta llegar a la idea de que enseñar y aprender se aproximaron en un vínculo indisoluble y dejaron que la didáctica se convierta en una ciencia de la enseñanza y de la mediación, no en una ciencia del sujeto (véase Holzkamp 1993)-. Asimismo, sería interesante buscar el concepto de aprendizaje en rumano e investigar qué visiones didácticas del mundo originan p. ej. las palabras rumanas «invat(z)are» [aprender] y «predare» [enseñar]. «Predare» [depredar] y «Predigen» [predicar] se remontan a la misma raíz y probablemente también están de alguna manera relacionadas con la palabra latina para la «presa» ${ }^{4}$. Mientras que aprendo, capturo algo, aunque lo apresado, el botín ${ }^{5}$ siempre señala también un tipo de toma de posesión ilícita, una apropiación, por así decir, sin derecho; asimismo, esta es una connotación que hace alusión al poder y al derecho y continúa teniendo un efecto en la visión didáctica del mundo.

¿Se trataba del poder social que quería controlar y limitar el aprendizaje autónomo? ¿Se intentaba que se establecieran los asuntos del control de la iglesia y de las autoridades, mediante la disciplina del «cuerpo dócil», como lo expresó Michel Foucault? Son esos los motivos por los que finalmente desde el término del aprendizaje se generó el de enseñanza y permitieron el cambio de un vínculo educativo a una «desproporción educativa», como constató Nora Sternfeld, austríaca y profesora en la Universidad Aalto en Helsinki, en conexión con Rancière, Gramski y Foucault. Sobre Foucault escribe lo siguiente:

"En la genealogía de los mecanismos disciplinarios los miembros de la sociedad parecen ser solo seres condicionados. ¿En qué medida "Vigilar y castigar" se refiere a una perspectiva puramente determinista de la sociedad?». (Sternfeld 2009: 97).

Pero ¿esta mirada determinista, que se hizo concebible mediante la dualidad de enseñanza y aprendizaje, es realista? ¿O proviene de una ilusión de manejabilidad de una situación en la cual -realizando una valoración sobria- no hay nada para controlar?

Recordemos lo siguiente: «Si los hechos cambian, cambio mi opinión. Y usted, ¿qué hace?»-esta es la pregunta de Keynes. Esta pregunta se dirige directamente a nosotros mismos que hemos sido socializados en contextos de enseñanza y aprendizaje y a nosotros que incluso nos consideramos formadores y

\footnotetext{
Veáse www.h-age.net/hinter-den-kulissen/144-was-ist-lernen-etymologische-wurzeln-definitionen.html (consulta el 13.4.2017).

4 En castellano esta relación se encuentra en aprender/aprehender [N. trad.]

5 En alemán, erbeuten es apresar y los apresado, Beute, es tanto la presa como el botín, noción esta que tiene una connotación de ilicitud. [N. trad.]
} 
que también queremos seguir considerándonos así. ¿ Somos realmente capaces de desistir de valoraciones apreciadas cuando los análisis objetivos nos enseñen algo mejor? ¿Qué tipo de sentimientos nos acechan cuando debemos darnos cuenta de que estábamos equivocados? ¿Nos corregimos a nosotros mismos o insistimos al esforzarnos en conservar, organizar una parte de nuestras convicciones actuales sin saber de qué manera y en enseñar dónde en realidad solo se puede aprender?

\section{El futuro como continuidad: la anticipación de aplicaciones posteriores}

Hasta el día de hoy, la idea de la capacidad de anticipación a desafíos futuros cumple un papel fundamental para el pensamiento pedagógico. Si bien uno es consciente que en realidad ninguna persona puede prever el futuro, es de utilidad valerse de la aceptación general de que todo no será muy diferente a como lo es hoy en día. Esta presunción puede haber sido verdad durante mucho tiempo; pero en períodos de innovaciones disruptivas pierde casi por completo su razón (Véase Christensen, 2011). La esencia de estas innovaciones es justamente que no resultan de las prácticas anteriores sino que a menudo se infiltran de otras áreas a través de efectos transversales. Por ejemplo, no se trata de que un fabricante fotográfico más competente -analógico- que revolucione la tecnología preferida en esta área revoluciona y desplace al actual líder del mercado, sino que una tecnología digital perfeccionada y utilizada en otro sitio (p. ej. en Silicon Valley) se imponga sin preámbulos ni consideraciones como una alternativa inesperada y de gran rendimiento. Este fue el destino de la empresa Kodak -en un tiempo la empresa de ingeniería fotográfica más grande del mundo que, gracias a la fotografía digital, fue casi completamente arrasada y estuvo a punto de quedar en la ruina. Asimismo, en el sector de taxis y transporte Google amenaza con interponerse entre los actuales actuar en el negocio básico-.

¿Cómo puede ser una anticipación de situaciones que se apliquen posteriormente, si la disrupción reemplaza a la innovación? ¿Cómo se prepara a los aprendices, estudiantes universitarios y adultos para enfrentar las disrupciones de los mercados de trabajo del futuro?

Este tipo de cuestiones ponen en tela de juicio las ideas tradicionales. No podemos seguir manejándonos en el -supuesto- terreno seguro de lo especializado y calculable, sino que debemos aprender a extraer de la inseguridad y la apertura al futuro las consecuencias correctas a nivel educativo, curricular y, en especial, didáctico. Esto no es nada fácil, ya que el nivel alcanzado profesionalmente de los productos y servicios también continuará siendo el margen fundamental de competitividad: Todos adquirimos el móvil con el mejor diseño y de muy fácil uso sin preguntar por las relaciones de producción y cualificación, a las que les agradecemos estas ventajas en la comparación de mercados. La superioridad técnica ya no resulta solo de la profesionalidad de los agentes implicados sino de una conectividad mundial en el diseño de productos. En términos sencillos, se puede afirmar lo siguiente: La concentración de todas las competencias profesionales en una sola persona - el o la profesional en el mundo digital interconectado será reemplazada por la combinación de redes y el uso de especializaciones distribuidas y ventajas competitivas.

No la permanencia del «humanismo especializado» [Fachmenschentum] (Max Weber) sino su fragmentación, la desfronterización y la desprofesionalización parecen ser las señales del futuro. Además, todos nos enfrentamos la amenaza de «la trampa de la continuidad». Esta trampa nos seduce y nos conduce a un conservadurismo involuntario que, al fin y al cabo, se aferra a la hipótesis de que, en líneas generales, también el futuro será como lo ocurrido anteriormente. No obstante, este efecto contribuye asimismo a que una y otra vez nos encontremos en una situación dónde queramos solucionar problemas con las «maneras de pensar» con las cuales a la vez también causamos los problemas - una autolimitación del tipo 
trascendental de la cual ya Albert Einstein (1879-1955) sabía decir que aquellas maneras nunca podrían funcionar realmente (véase Stahlbaum, 2014)-.

\section{El futuro como salida: la anticipación del todavía no}

Las sugerencias para repensar el futuro se encuentra también y justamente en aquellos que han fracasado ante las formas de educación dadas y que tuvieron que descubrir por sí mismos nuevos caminos -en la mayoría de los casos informales- para llegar a su propia capacidad de aprendizaje - esto se encuentra en las ofertas de apoyo de la educación integradora o incluso terapéutica (véase Kreszmeier 1994), así como en los enfoques referidos al fortalecimiento de la personalidad de la formación profesional y también en las concepciones europeas acerca de una formación permanente-. Es importante mencionar, en especial, la situación en la que el aprendizaje de los adultos se pudo desarrollar a través de varias décadas solo a la sombra del espíritu de una época que estaba caracterizada por la siguiente frase «Lo que Juanito no aprende, ya nunca lo aprenderá Juan». Paulatinamente se amplió la mirada hacia la lucha permanente de los adultos por la identidad y la competencia y se abrió paso una perspectiva que la científica cognitiva suiza Elsbeth Stern resumió en la frase contraria: «Lo que Juanito no aprende, lo aprenderá Juan después» (Stern, 2011: 93 y ss.).

Al mismo tiempo, se concedió también más importancia a la orientación global así como al referente de identidad, y a la informalidad del aprendizaje de los seres humanos. El aprendizaje de los adultos se redefinió como «un proceso cognitivo relacionado con el mundo de vida» (Schmitz, 1984) y uno se desempeñaba en formas de un aprendizaje desde el otro. Esta perspectiva, que desde los años 90 se amplió hasta los conceptos constructivistas y sistémicos de la formación de adultos (véase Arnold/Siebert, 2006; Arnold, 2013), se anticipó a lo que han clarificado de manera creciente e ineludible las investigaciones del cerebro sobre la adquisición de nuevos conocimientos desde principios de siglo. Un neurocientífico resume los resultados de 2016 con las siguientes palabras:

«La dimensión de los cambios ocasionados en el interior depende siempre de qué tipo de patrones de reacción y respuestas disponga ya el ser vivo en cuestión y con qué grado de eficiencia dicho ser vivo puede activar y emplear los patrones. Y eso, a su vez, depende de las respectivas experiencias previas, que ya pudo realizar para resolver problemas similares y desafios y pudo establecer en su interior como patrones adecuados de respuestas y reacciones».

«Entonces, las experiencias previas son decisivas para saber si cambios determinados que se producen en el mundo exterior o en el interior de un ser viviente se deben experimentar y valorar como algo significativo -y si aqui se desencadena su propio proceso de aprendizaje-. [...]. Todos los seres vivos desarrollan sus respectivos patrones de reacción y respuestas cimentados estructuralmente sobre la base de las soluciones encontradas por ellos mismos en el transcurso de su desarrollo previo. Estas soluciones encontradas por ellos son relevantes. No son objetivamente o igualmente importantes para todos, sino solo lo son siempre para el ser vivo en cuestión. Por ello, todos los procesos de aprendizaje se caracterizan por la atribución subjetiva de significatividad. Y por consiguiente, tampoco se puede aprender lo que no tiene sentido para un ser viviente». (Hüther, 2016, pág. 45).

Estos conocimientos obtenidos a través de la investigación de las ciencias naturales refuerzan otra mirada hacia los procesos de enseñanza y aprendizaje. Centran la atención en la lógica de la apropiación como movimientos de apoyo de procesos subjetivos de transformación y, así, comparten una visión que resulta familiar desde tiempos inmemoriales a la práctica educativa de adultos y a su teoría. El yo de su 
mundo de vida encuentra de algún modo su anclaje en las ciencias naturales en el «yo sináptico» (LeDoux 2002). Ambos tienen en común el discernimiento inevitable de que es necesario superar la enseñanza y las concepciones del control de aportes (inputs) para poder estimular, acompañar y apoyar de manera más eficaz el desarrollo del aprendizaje y las competencias en su lógica dentro-fuera (inside-out). El programa didáctico para el desarrollo de competencias y la política educativa de la UE tiene el mismo hilo conductor: dónde había un aporte (input), tiene que haber un resultado (outcome).

Este todavía-no de la educación se tiene que entender en primer lugar como un esbozo. Si se observa atentamente el discurso educativo en Europa, no se puede pasar por alto que la exigencia de preparación de las instituciones educativas ya se ha visto seriamente afectada. Si hay algo de cierto en el pronóstico de Ray Kurzweil, según el cual experimentaremos en el siglo xxi un cambio de las condiciones de vida, requisitos y posibilidades del ser humano que corresponden en su intensidad quizás a la transformación de la historia de la humanidad de los 20.000 años pasados, entonces debemos modificar con urgencia nuestro concepción educativo «aprender del pasado». Así debemos desligarnos de la fijación de contenidos curriculares para fortalecer de tal manera a las nuevas generaciones como personalidades para que en realidad sean capaces de «llevar a cabo nuevas situaciones de manera autodirigida y apropiada»-como afirma, por ejemplo, la definición del término de la competencia del Marco Europeo de Cualificaciones-. Teóricos destacados de la educación reconocieron hace ya mucho que esta demanda corresponde al concepto de una teoría educativa formal que trata de aclarar más exhaustivamente la siguiente pregunta: de hecho, cómo se pueden iniciar y fomentar dichas competencias en los sujetos. Quién es capaz de reconocer solo la desaparición de conceptos probados en dichos desarrollos (Liessmann, 2016; Türcke, 2016), ignora y banaliza no solo eso sino que también rechaza la relación de evidencia reclamada por Keynes: «Si los hechos cambian, cambio mi opinión. Y usted, ¿qué hace?». Ignorar las evidencias hace que la política educativa vuelva a la situación de «sigamos así como hasta ahora», que a largo plazo ya no será capaz de convencer a nadie.

\section{Futuro como disolución: la contemplación de lo biográfico}

¿Qué sabemos acerca del fomento y formación de competencias para configuración autogestionada de nuevas situaciones de requerimientos aún no previsibles? También en esta cuestión se encuentra hasta ahora una escasa orientación en el discurso de la pedagogía europea que vaya más allá de un «sigamos así». Es alarmante cómo en los debates se observan los efectos reales de la práctica educativa actual de manera tan poco crítica. Tampoco se analiza la sostenibilidad escandalosamente baja del aprendizaje actual en vías curriculares en las cuales se pierden a menudo casi por completo los conocimientos de muchos años escolares, ni tampoco tiene lugar un verdadero análisis de los resultados de los neurocientíficos que nos reclaman al unísono:

«Proporcionar contenidos o incluso competencias no funciona, incluso si nos representamos y nos aferramos a ellos, en lugar de disolver los modelos establecidos!».

Sus hechos apuntan a la configuración necesaria de contextos para la adquisición autoorganizada de contenidos, en cuyo caso la cuestión no se centra tanto en el control o instrucción mediante el personal docente sino más bien en el acompañamiento y asesoramiento de procesos de búsqueda. Se hace evidente lo siguiente: Debemos consolidar el concepto de una «educación multidimensional», como lo solicita un nuevo memorándum con el título programático «Educación. Mucho más que la profesionalidad» (Vereinigung, 
2015). En dicha «educación multidimensional» además de las competencias específicas, se debe tener como objetivo fortalecer «la estructura de la personalidad, la seguridad conductual y la formación del carácter de los jóvenes». Esto requiere profesionalidad por parte de los responsables, la cual se describe más apropiadamente con el término «orientación pedagógica» que con las propuestas unidimensionales y retrógradas de aquellos que eluden el llamamiento de Keynes.

¿Qué requisitos están asociados a dichas rupturas de patrones para el rol de los docentes pero también para los padres, pedagogos o formadores en el proceso del aprendizaje o la formación permanente? Queda claro lo siguiente: Necesitamos un concepto de aprendizaje contemporáneo. El aprendizaje no se puede justificar más, en particular, como consecuencia de la enseñanza. Por el contrario, en los últimos años los neurocientíficos y los pedagogos se concentran en el sujeto desarrollado y hacen referencia al ser humano como «el animal que es capaz de aprender», que ya siempre estaba en condiciones de manejarse de manera autoorganizada y creativa con las indicaciones de su entorno - de hecho, desde hace al menos 400.000 años y no desde que existen docentes (primera aparición de forma aislada aproximadamente 2000 antes de Cristo).

La pregunta sobre el futuro no puede ocultar con carácter permanente la propia delimitación temporal y la pregunta «¿qué es el ser humano?»-un pensamiento profundo y tradicional de la pedagogía pero que también se ha tornado impopular-. Sin embargo, antes del no-yo, es decir, de la pérdida o la falta del yo se abre una dimensión hacia un enfoque explicativo que es capaz de transportarnos hasta nosotros mismos. No somos lo que pensamos y tampoco debemos permanecer en la cuestión de como hemos llegado a ser lo que somos - estas son las indicaciones de este «giro contemplativo», tal como han sido perseguidas por Francisco Varela y algunos representantes de la pedagogía norteamericana-. De esa manera, diseñan también un plan de una forma de proceder de manera reconocida y configuradora de la realidad -de la del propio interior y del supuesto exterior- y, asimismo, señalan otra imagen de futuro del discurso pedagógico. Por último, su «contemplative approach» tiene como finalidad:

\section{«Desarrollar la habilidad de ser capaz, de observar claramente y utilizar la propia subjetividad de forma imparciali. (Roth 2009, pág. 102).}

Este enfoque deja atrás el enfoque-de-tercera-persona de la observación cotidiana, aunque también de la científica, con el cual miramos el mundo centrados en el objeto y hablamos sobre él en nuestras lenguajes arbitrarios. En su lugar, el enfoque-de-primera-persona de la observación atenta ocupa un lugar más importante, como, entre otros, fue impulsado a través de la fenomenología de Husserl y Merleau-Ponty aunque también fue fomentado por concepciones budistas o ecológicas (véase Karafilidis, 2016: 227 y ss.). En esta forma de la observación, del modo de hablar y actuar, el uso de la lengua permanece anclado en una lógica reflexiva, que puede acompañarse de la autorreferencia como autocrítica. Los investigadores contemplativos son conscientes de la frase a menudo citada de Ludwig Wittgenstein «De que me parezca a mí -o a cualquiera- que sea así, no se sigue que es así» (Wittgenstein, 1984: 119), por lo que cada vez más están capacitados para «manejar» los mecanismos de su cognición y su percepción lingüística. En este sentido, Francisco Varela, entre otros, habla del «manejo de la cognición» («bandle of cognition») de manera consciente y experimentada, en el cual los actores constantemente perciben, juzgan e interactúan en su consciente como, por último, banal y transparente funcionan -en definitiva de manera banal y transparente- en nosotros mismos la percepción, el juicio y la lengua (véase Depraz/Varela/Vermersch, 2002: 155 y ss.). Los seres humanos pueden reconocer que quedan por detrás de sus posibilidades y que sus 
propias vidas obedecen a la repetición de patrones incomprendidos. El «sapere aude!» [atrévete a saber] de Immanuel Kant es también un llamamiento a la reflexión y transformación de estos patrones, o bien, el llamamiento a romper con los patrones - una demanda para profundizar el propio acceso al mundo que poco tiene que ver con promesa de ascenso de la pedagogía moderna-.

En las concepciones educativas autoreflexivas y contemplativas están adquiriendo más importancia tal vez las dimensiones de una formación de la personalidad y la actitud, tal como se contraponen o son puestas a un lado de las teorías educativas materiales, por lo menos desde la teoría de Wilhelm von Humboldt. En ese tipo de formación de personalidad se trata de

- fortalecer las fuerzas-del-yo y los potenciales de cada uno,

- fomentar su toma de posición justificada de lo que realmente significa la vida y

- desarrollar continuamente sus competencias de autoformación y de autoaprendizaje, como se suele decir ahora. Aquellas, si bien necesitan el acceso social a los contextos de apoyo, también requieren la integración emocional en experiencias de reconocimiento y autoeficacia y en contextos de lo vivenciado.

En ese tipo de formación se trata menos de la provisión de conocimientos seguros que del fomento de una actitud interna que sea capaz de cuestionar las certezas propias y que de forma permanente trate de encontrar soluciones adecuadas y viables. Esa especie de educación y actitud presupone también capacidades contemplativas del individuo en el trato consigo mismo y con el mundo, como p.ej. las capacidades

- para prestar total atención sin influencias distorsionantes de los propios concepciones,

- para el aplazamiento de las propias suposiciones y apreciaciones así como para su comprensión,

- para la profunda compenetración con otros, con sus condiciones y circunstancias,

- para tratar de comprender y sentir compasión así como respeto en la forma de vivir y opiniones de otras personas,

- para la familiaridad y la intimidad,

- para el incremento de percepción de relaciones holísticas e integradas de causa a efecto así como

- para la participación profunda y comprometida en interacciones con otros (véase Gunnlaugson et al., 2014: 5).

La formación sostenible de dichas capacidades tiene que ver más con las propios rastros emocionales de los seres humanos en sus fases biográficas de desarrollo que con los contenidos de los planes de estudio y currículums. En fases más avanzadas del desarrollo, las huellas biográficas se pueden socializar posteriormente a través de la autorreflexión y los ensayos que la acompañan, sin embargo, las propias formas iniciales del trato de sí mismo y del mundo en raras ocasiones pueden ser superadas completamente. En cualquier caso, dicha formación de actitud supone un aprendizaje reflexivo que suscite la búsqueda y el autoconocimiento, ya que en definitiva ambos determinan sutilmente el modo en que se lleva a cabo el trato con los saberes.

La persona que no pudo desarrollar las llamadas capacidades contemplativas tiende a tener una visión del mundo que cree describir a los otros seres humanos y al mundo de manera «objetiva» y, de tal forma, poder dominarlo técnicamente. En cambio, una formación autoreflexiva y contemplativa propicia la 
formación de una consciencia que es capaz de ver en otros conceptos y patrones de conducta también la expresión de una búsqueda humana. Los intelectuales contemplativos nunca preguntan quién tiene razón, ellos únicamente se esfuerzan por reconocer en ellos mismos y en los demás los patrones del comportamiento consigo mismos y el mundo, para mejorar así la conectividad recíproca. Por ello, son maestros en la búsqueda, pero no en el hallazgo. Tampoco son buenos en las discusiones para determinar quién tiene la razón. Más bien buscan en el conocimiento de Sócrates que dijo: «solo sé que no sé nada». Solo la persona supuestamente conocedora espera de un incremento en el saber un aumento de sus posibilidades; por el contrario, el no conocedor distingue el escepticismo frente al impacto rígido de sus verdades que es capaz de colocarlo en trance lo que le impide realizar otra búsqueda.

Por lo tanto, la formación de la personalidad no es solo una palabra sino un programa -y ciertamente unos especial-. Allí la idea logra la expresión de que el ser humano pueda emprender su propio viaje para llegar a ser lo que él o ella pueda se (véase Arnold, 2016). Esta formulación puede que suene poco clara y también ambiciosa y que describa mucho más un compromiso continuo que un resultado favorable, pero al menos se centra claramente en el aspecto de la autoformación - un movimiento que se sostiene por el activo interés para llegar a saber «cómo se ve al mundo a través de otros ojos» y cómo se puede conseguir «ampliar el propio campo visual de esa manera» (Spaemann, 1994/95: 34)-. Este cambio de perspectiva también ocupa un lugar preponderante en el concepto de la libertad, tal como lo propugnó en su discurso Carolin Emcke, galardonada con el Premio de la Paz de la industria del libro alemana en 2016. Está claro que la educación no se puede concebir sin libertad, como también la libertad requiere educación. Emcke afirmó lo siguiente:

«No podemos mantenernos solo como una sociedad democrática de secularización libre sino que también debemos serlo. La libertad no es algo que uno posea, más bien es algo que uno bace. La secularización no es una cosa terminada sino un proyecto inconcluso. La democracia no es una seguridad estática, sino más bien una práctica dinámica en la gestión de la incertidumbre y la crítica. Una sociedad libre, secular y democrática es algo que debemos aprender. Una y otra vez. Al escuchar unos a otros. Al reflexionar sobre los demás. En el diálogo y la acción conjunta. En el respeto mutuo de la diversidad de afiliaciones y la singularidad individual. Y no menos importante, en el reconocimiento muto de debilidades y en el perdón».

"iEs un trabajo arduo? Si, totalmente. ¿Se crearán conflictos entre las diferentes prácticas y convicciones? Si, seguramente. ¿Será a veces difícil encontrar un equilibrio justo entre las referencias religiosas respectivas y el orden secular? Absolutamente. Pero ¿̇or qué debería ser también fácil? Siempre podemos volver a comenzar. ¿Qué se necesita? No mucho: un poco de actitud, otro poco de valor risueño y nada menos que la disposición de cambiar la perspectiva para que ocurra con más frecuencia y que todos digamos: Wow. Asi se ve el panorama desde esta perspectiva». (Emcke, 2016).

Este tipo de discurso autoreflexivo y contemplativo no es algo nuevo, aunque no es muy practicado. Si bien es cierto que las teorías autoreflexivas así como el conocimiento para los mecanismos ligados al lenguaje de nuestra percepción no han sido elaborados recientemente, también es cierto que a pesar de las numerosas referencias de la filosofía del lenguaje así como de la investigación cognitiva, del cerebro y de la meditación en nuestra vida cotidiana, profesional y privada, la mayor parte del tiempo nosotros hacemos como si aquellas no tuviesen ningún significado para nuestro pensamiento, nuestros sentimientos, nuestra habla o nuestra acción. El arte de manejar de forma sensata los mecanismos transparentes de nuestra cognición y emoción aún no está difundido. Este arte nos puede ayudar a repensar y experi- 
mentar nuestras posibilidades biográficas con nuevos términos y de una manera fuertemente desligada de nuestras experiencias. La adquisición y la práctica de este arte es un «trabajo interno». Practicarlo puede conducirnos a nuevas formas de reflexión que investiguen los propios mecanismos del manejo de lo vivo en nosotros mismos - un aspecto de la formación futura que solo se puede aprehender en este enfoque (véase Arnold, 2017)-. Este será la formación de la personalidad en un sentido tanto reflexivo como transformador -completamente en el sentido de una definición fundamental de la educación de personas adultas de Alemania, que fue definida ya tempranamente como «el esfuerzo permanente de comprenderse a sí mismo y al mundo y actuar conforme a este conocimiento» (véase Arnold; Nuissl; Rohs, 2017)-.

\section{Referencias bibliográficas}

Arnold, Rolf (2013). Systemische Erwachsenenbildung. Die transformierende Kraft des begleiteten Selbstlernens. Baltmannsweiler.

Arnold, Rolf (2017). Es ist später als du denkst! Perspektiven für die Restbiografie. Berna: Hep

Arnold, Rolf; Erpenbeck, J. (2015). Wissen ist keine Kompetenz: Dialoge zur Kompetenzreifung. Baltmannsweiler.

Arnold, Rolf; Siebert, H. (2006). Konstruktivistische Erwachsenenbildung. Von der Deutung zur Konstruktion von Wirklichkeit. $4^{a}$ edición. Baltmannsweiler.

Arnold, Rolf; Nuissl, Ekkehard; Rohs, Matthias (2017). Erwachsenenbildung. Baltmannsweiler.

Burow, O. F. (2016): “Creative Collaboration: Schlüsselkompetenz für LehrerInnen und Schüler/innen im Digitalen Zeitalter”, Journal für Lebrerbildung, 1, pp. 7-16.

Christensen, Clayton M. (2011). The Innovators Dilemma. Warum etablierte Unternebmen den Wettbewerb um bahnbrechende Innovationen verlieren. Múnich: Vahlen.

Depraz, Nathalie; Varela, Francisco J.; Vermersch, Pierre (eds.) (2002). On Becoming Aware. A pragmatics of experiencing. Amsterdam: John Benjamins.

Drees, Gerhard (2001): "Was ist Persönlichkeitsbildung im Beruf? - Konzeptionelle Reflexionen zu einem fast vergessenen Anspruch" en Holger Reinisch, Reinhard Bader, Gerald A. Straka (eds.): Modernisierung der Berufsbildung in Europa. Neue Befunde der berufs- und wirtschaftspädagogischen Forschung. Wiesbaden: Schriften der DGfE.

Emcke, Carolin (2016): “Die Populisten und Fanatiker hassen nicht unbedingt selbst - sie lassen hassen”, Süddeutsche Zeitung, 23 de octubre.

Erpenbeck, John; Sauter, Werner (2016). Stoppt die Kompetenzkeatastrophe. Wege in eine neue Bildungswelt. Wiesbaden.

Erpenbeck, John; Sauter, Werner (2013). So werden wir lernen. Kompetenzentwicklung in einer Welt füblender Computer, kluger Wolken und sinnsuchender Netze. Wiesbaden.

Foucault, Michel (1976). Überwachen und Strafen. Die Geburt des Gefängnisses. Fráncfort: Suhrkamp

Gunnlaugson, Olen; Sarath, Edward W.; Scott, Charles: Heeson, Bai (2014): “An Introduction to Contemplative Learning and Inquiry" en Olen Gunnlaugson, Edward W. Sarath, Charles Scott, Bai Heeson (eds.): Contemplative Learning and Inquiry across Disciplines. Nueva York.

Holzkamp, Klaus (1993). Lernen. Ein subjektwissenschaftlicher Ansatz: Stuttgart. 
Hüther, Gerald (2016). Mit Freude Lernen - ein Leben lang. Weshalb wir ein neues Verständnis vom Lernen brauchen. Gotinga: V\&R.

Jackson, Tony (2011): “Kodak fell victim to disruptive technology” en www.ft.com/cms/s/0/f49cb408ecd8-11e0-be97-00144feab49a.html\#axzz4lo0SBHrO. (2.10.2011)

Karafilidis, A. (2016): "Unmittelbares Handeln und die Sensometorik der Situation. Über Francisco Varela, Ethical Know-How (1992)" en Dirk Baecker (ed.): Schlüsselwerke der Systemtheorie. $2^{a}$ ed. Wiesbaden: Vs Verlag für Sozialwissenschaften.

Kreszmeier, Astrid Habiba. (1994). Das Schiff Noah. Dokumente einer therapeutischen Reise. Graz: Bibliothek der Provinz.

LeDoux, Joseph (2002). Synaptic Self: How Our Brains Become who We are. Londres:Viking.

Liessmann, Konrad Paul (2016). Geisterstunde. Die Praxis der Unbildung. Eine Streitschrift. Múnich: Zsolnay.

Myngyur, Yongey R. (2007). Buddha und die Wissenschaft vom Glück. 2a ed. Múnich: Goldmann

Rauning, Gerald; Wuggenig, Ulf (eds.) (2007). Die Kritik der Kreativität. Viena: Turia + Kant

Riemer, Friedrich Wilhelm (1819). Griechisch-Deutsches Handwörterbuch für Anfänger und Freunde der griechischen Sprache. $3^{a}$ ed. actualizada. Jena, Leipzig.

Roth, Gerhard (2007). Persönlichkeit, Entscheidung und Verbalten. Warum es so schwierig ist, sich und andere zu verändern. Stuttgart: Klett-Cota.

Roth, Harold D. (2014): "A Pedagogy for the New Field of Contemplative Studies" en Olen Gunnlaugson, Edward W Sarath, Charles Scott, Bai Heeson (ed.): Contemplative Learning and Inquiry across Disciplines. Nueva York: State University of New York.

Ruhloff, Jörg (1996): “Bildung im problematisierenden Vernunftgebrauch” en Michele Borelli, Jörg Ruhloff, (eds.): Deutsche Gegenwartspädagogik. Tomo II. Baltmannsweiler: Schneider Hohengehren.

Schmidt, Franz; Stäckel, Paul (eds.) (1899). Briefwechsel zwischen Carl Friedrich Gauss und Wolfgang Bolyai. Leipzig: Teubner.

Schmitz, E. (1984): "Erwachsenenbildung als lebensweltbezogener Erkenntnisprozess", Enæyyklopädie Erziehungswissenschaft. Tomo XI. Stuttgart, pp. 95-123.

Spaemann, Robert (1994/1995): "Wer ist ein gebildeter Mensch?”, Scheideweg. Jahresschrift für skeptisches Denken, XXIV, pp. 34-37.

Spitzer, Manfred (2007). Lernen, Gehirnforschung und die Schule des Lebens. Múnich: Spektrum Akademischer Verlag.

Sternfeld, Nora (2009). Das pädagogische Unverbältnis. Lebren und lernen bei Rancière, Gramsi und Foucault. Viena: Turia + Kant.

Stahlbaum, Dietrich (2014): "Probleme kann man niemals mit derselben Denkweise lösen, durch die sie entstanden sind”, Zeitkritische Beiträge. Múnich. 
Stern, Elsbeth (2006): "Was Hänschen nicht lernt, lernt Hans hinterher. Der Erwerb geistiger Kompetenzen bei Kindern und Erwachsenen aus kognitionspsychologischer Perspektive" en Ekkehard Nuissl (ed.): Vom Lernen zum Lehren: Lern- und Lehrforscbung für die Weiterbildung. Bielefeld: Bertelsmann.

Türcke, Christoph (2016). Lehrerdämmerung. Was die neue Lernkultur in den Schulen anrichtet. Múnich: C.H.Beck. Vereinigung der Bayerischen Wirtschaft e.V. (ed.) (2015): Bildung. Mehr als Fachlichkeit. Gutachten. Münster.

Wittgenstein, Ludwig (1984): Über Gewissheit. Werkausgabe. Tomo VIII: Bemerkungen über die Farben. Über Gewissheit. Zettel. Vermischte Bemerkungen. Fráncfort d. M: Bibliothek Suhrkamp.

\section{Nota biográfica}

Rolf Arnold es catedrático de Pedagogía de la Universidad Técnica de Kaiserslautern (Alemania). Especialista en formación continua y formación de personas adultas. Es responsable de Campus Virtual de Renania-Palatinado. Su posición teórica, que se define como «Didáctica posibilitante», combina el constructivismo emocional con la pedagogía sistémica. 